\title{
Prevalencia de ideación e intento suicida en adolescentes de la Región Metropolitana de Santiago de Chile
}

\author{
RAÚL VENTURA-JUNCÁ D. ${ }^{1}$, CÉSAR CARVAJAL², SEBASTIÁN UNDURRAGAa \\ PILAR VICUÑA ${ }^{\mathrm{a}}$, JOSEFINA EGAÑA ${ }^{\mathrm{a}}$, MARÍA JOSÉ GARIB ${ }^{\mathrm{a}}$
}

\section{Prevalence of suicidal ideations and suicidal attempts among adolescents living in Metropolitan Santiago}

\begin{abstract}
Background: According to World Health Organization (WHO) estimates, suicide is one of the five leading causes of death among people aged 15-24 years. Suicide rates in Chile among young people have been increasing. In 1990 and 2005, the prevalence of suicide was $8.8 \%$ and $9.3 \%$ per 100,000 inhabitants, respectively. Aims: To determine the prevalence of suicidal ideation and suicide attempt rates among teenagers aged 14 and 19 years, living in Metropolitan Santiago and analyze the factors related to suicide. Material and Methods: A self administered enquiry, containing a depression scale and five questions about suicide tendency, was applied to a representative sample of teenagers aged between 14 and 19 years, attending public and private schools in Metropolitan Santiago. Results: The enquiry was answered by 1567 teenagers aged between 14 and 20 years (54\% males). Sixty two percent recognized suicidal ideation and 19\%, suicidal attempts. These figures were significantly higher among women and those attending public schools. Sixteen years old adolescents had the highest rates of suicidal ideation and attempts. Conclusions: There is a high rate of suicidal ideation and attempts among teenagers living in Metropolitan Santiago.
\end{abstract}

(Rev Med Chile 2010; 138: 309-315).

Key words: Adolescent; Chile; Suicide, attempted.

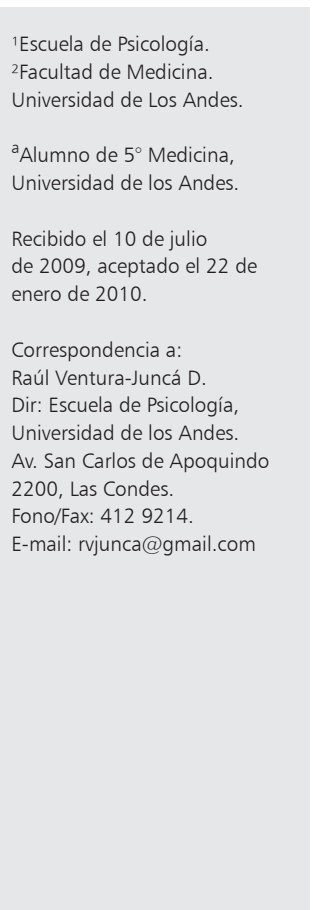

L a Organización Mundial de la Salud (OMS) establece el suicidio dentro de las cinco principales causas de mortalidad entre los 15 y 19 años ${ }^{1}$. La frecuencia mundial de suicidio e intentos suicidas en adolescentes entre 1970 y 1990, muestra comportamientos disímiles entre los distintos países. Mientras que en naciones como Irlanda, Israel y Noruega hubo un crecimiento explosivo, en Yugoslavia y Suecia disminuyó ${ }^{2}$. En las últimas décadas los países de Europa del Este muestran la mayor incidencia anual de suicidio consumado ${ }^{3}$.

En Chile, las tasas de suicidio en jóvenes entre
15 y 24 años han ido en aumento. La tasa de suicidio para este rango etáreo de la Región Metropolitana (RM) fue de 8,83 por 100.000 habitantes el año 1990 y de 9,28 por 100.000 habitantes el año 2005 (Estadísticas suicidio 1990-2005. Servicio Médico Legal de Santiago. Chile). Sin embargo, se debe considerar que los registros de muerte por suicidio están subestimados, ya que dentro de la tasa de muerte por causa indeterminada debe existir un grupo importante que pertenece a suicidas ${ }^{4}$.

Algunas unidades de psiquiatría en distintas 
regiones de Chile han realizado importantes investigaciones acerca del suicidio adolescente. Salvo et al, en un trabajo sobre ideación suicida e intento suicida en adolescentes de enseñanza media de Concepción, observa que $57 \%$ de los jóvenes presenta ideación suicida y 14,2\% intento suicida a lo largo de su vida, con una prevalencia de suicidalidad de $71,2 \%$.

El estudio de Haquim et al, acerca de factores protectores y de riesgo en salud mental en niños y adolescentes de la ciudad de Calama, muestra un porcentaje de ideación entre 6,4 y $14,6 \%$, y $18 \%$ de uno o más intentos de suicidio ${ }^{6}$.

En cuanto a la casuística extranjera destacan dos estudios sobre comportamiento suicida en adolescentes: uno canadiense $\mathrm{e}^{7}$ y otro norteamericano $^{8}$. Éstos presentan $33 \%$ y $24 \%$ de ideación suicida y $9,3 \%$ y $3,3 \%$ de intentos, respectivamente. Una reciente revisión sistemática concluye que $29,9 \%$ de adolescentes ha tenido ideas suicidas en algún momento de su vida y $9,7 \%$ y $6,8 \%$ han atentado contra sí mismos alguna vez en la vida o en los últimos 12 meses, respectivamente ${ }^{9}$. En relación a los datos biodemográficos hay un predominio de suicidios e intentos de suicidios en sexo femenino, con un incremento a temprana edad, entre los 16 y 18 años $^{10-11}$. En cuanto a las desventajas socioeconómicas, éstas muestran escasa evidencia de asociación al intento suicida. Lo que sí se observa es que los adolescentes que intentan quitarse la vida provienen de familias más disfuncionales en comparación con adolescentes controles ${ }^{12}$.

El objetivo de este trabajo consiste en determinar la prevalencia de ideación e intento suicida en adolescentes entre 14 y 19 años en una muestra representativa de la Región Metropolitana (RM). Asimismo busca analizar la asociación entre sexo, edad y tipo de colegio versus ideación e intento suicida, para así poder contar con información reciente acerca de la suicidalidad en este grupo etáreo. Este objetivo busca fomentar la implementación de programas preventivos, tal como se ha hecho en Argentina, país que tiene la mayor tasa de suicidios adolescentes de Latinoamérica ${ }^{13}$.

La valoración del riesgo de suicidio supone la evaluación de la presencia de ideas suicidas, la presencia de trastornos psiquiátricos y aquellos factores que están asociados con un mayor riesgo de suicidio ${ }^{14}$. Si bien es claro el aumento de la incidencia de psicopatología en la población infanto-juvenil de nuestro país, y su relevancia para la valoración del riesgo suicida ${ }^{15}$, este trabajo se limita a buscar casos de suicidalidad y no a investigar las causas gatillantes ni a establecer los factores de riesgos implicados ${ }^{16-17}$.

\section{Sujetos y Método}

El presente estudio se realizó entre septiembre y octubre de 2007, en cinco establecimientos educacionales elegidos como muestra aleatoria entre todos los colegios inscritos en la RM. Para contar con una muestra representativa se dividieron los establecimientos en tres grupos de acuerdo a su dependencia: colegios particulares privados, particulares subvencionados y municipales. Se tomaron como criterios de inclusión el que los establecimientos fueran mixtos, con más de 50 alumnos por nivel y tuvieran enseñanza media. La proporción de colegios fue: uno privado, dos subvencionados y dos municipales. Se evaluó a 1.590 alumnos de primero a cuarto medio que asistieron a clases el día de la aplicación de los instrumentos. Se utilizó una encuesta autoaplicada, anónima y voluntaria, la cual se dividía en dos partes. La primera contenía la escala de depresión de Zung y la segunda constaba de cinco preguntas para evaluar distintos niveles de suicidalidad. La escala de Zung ${ }^{18}$ fue desarrollada en 1965 y explora el componente somático conductual de la depresión y otorga un puntaje que permite discriminar entre "no depresión", “depresión leve”, "depresión moderada" y "depresión grave". Las preguntas fueron las siguientes: 1. ¿Has pensado alguna vez que la vida no vale la pena? (VNP); 2. ¿Has deseado alguna vez estar muerto? (DEM); 3. ¿Has pensado alguna vez terminar con tu vida? (PTV); 4. ¿Has intentado alguna vez terminar con tu vida? (IS); 5. ¿Alguna vez te has producido daño sin intención de terminar con tu vida? (PS). Las respuestas podían ser negativas, afirmativas para los últimos 12 meses, afirmativas para un período anterior a los últimos 12 meses o afirmativa para ambas (que corresponde a prevalencia de vida). Estas preguntas están adaptadas de la escala de Okasha $^{19-20}$ que utiliza las mismas preguntas pero con respuestas tipo Likert "nunca", "casi nunca", "algunas veces", "muchas veces".

Al momento de realizar la encuesta hubo al menos un investigador en la sala de clases para explicar la forma de contestarla y resolver las dudas. 
La encuesta duró aproximadamente 15 minutos. Se contó con la autorización de parte de los Directores de cada uno de los colegios. Se obtuvo el consentimiento informado pudiendo los alumnos abstenerse del estudio. Hubo seis alumnos que rechazaron participar en la investigación y 23 encuestas que fueron anuladas por estar contestadas incorrectamente.

El diseño de este trabajo fue de carácter transversal, retrospectivo, descriptivo e incluyó comparaciones con las distintas variables independientes. El análisis de los resultados se hizo con el programa estadístico informático SPSS versión 16.0 (Statistical Package for the Social Sciences). Para desarrollar el primer objetivo de este trabajo - determinar la prevalencia de ideación e intento suicida representativa de la RM-, se tomó una submuestra aleatoria estratificada y estandarizada con los mismos porcentajes de sexo, edad y tipo de colegio de la RM según los alumnos matriculados el año $2006(n=785)$. Para el segundo objetivo - estudiar la asociación entre sexo, edad y tipo de colegio versus ideación e intento suicida-, se analizó la información de dos formas distintas, con objeto de poder compararla y así disminuir al mínimo el error estadístico. La primera manera fue utilizando la muestra total obtenida estandarizada por sexo y tipo de colegio $(\mathrm{n}$ total ponderado $=$ 1.249) y la segunda fue utilizando una submuestra aleatoria y representativa de la muestra total pareada por edad, sexo y tipo de colegio $(n=480)$. Las dos formas de análisis de la muestra obtuvieron los mismos resultados. La prueba estadística utilizada fue el Chi Cuadrado de Pearson.

\section{Resultados}

\section{Prevalencia de ideación e intento suicida representativa de la RM en adolescentes entre 14 y 19 años.}

Para obtener la muestra representativa de la RM se utilizaron datos del MINEDUC (Ministerio de Educación de Chile) en cuanto a distribución de las matrículas escolares de la RM por dependencia, sexo y edad del año 2006, con el fin de obtener datos extrapolables a la región ${ }^{21}$. El 54,1\% de los encuestados eran hombres y $45,9 \%$, mujeres. Las edades fluctuaron entre los 14 y los 20 años, con un promedio de 16,2. Para simplificar la exposición de los resultados se agruparon las respuestas
VNP (Vida no vale la pena), DEM (Deseado estar muerto) y PTV (Pensado en terminar con su vida) en el ítem "ideación suicida". De este modo, la prevalencia de vida de ideación suicida que se obtuvo fue de $62 \%$, valor que triplica la prevalencia de intento suicidio (19\%), como lo muestra la Figura 1. Esta relación se mantiene al observar lo que ocurre en los últimos 12 meses, donde la ideación y el intento alcanzaron $45 \%$ y $11 \%$ respectivamente.

Se puede obtener una relación entre los niveles de suicidalidad a nivel de la RM (Figura 2), donde se puede extrapolar que de un total de 100 adolescentes de enseñanza media, 52 de ellos pensarán que la vida no vale la pena. De estos últimos, 42 desearán estar muertos, 32 pensarán terminar con su vida y 16 de los 52 lo intentarán. Es decir, por cada tres adolescentes de enseñanza media que piensan que la vida no vale la pena, dos piensan terminar con ella y uno lo intenta.

\section{Análisis de asociación entre sexo, edad y tipo de colegio versus ideación e intento suicida.}

En cuanto a las diferencias por sexo (Figura 3) se observó que las mujeres presentaban una prevalencia de vida de ideación suicida de $71 \%$, mientras que los hombres $49 \%$, diferencia estadísticamente significativa $(\mathrm{p}<0,001)$.

En las mujeres se observó una prevalencia de vida de intento suicida de $26 \%$ y en los hombres de $12 \%$, diferencia también estadísticamente significativa $(\mathrm{p}<0,001)$.

Para analizar la incidencia de ideas suicidas por edad (Figura 4) se utilizó el indicador de mayor gravedad de la ideación suicida, PTV, en los últimos 12 meses. Se observó que la mayor frecuencia de ideación suicida es a los 15 años (27\%), para luego disminuir proporcionalmente a medida que pasa el tiempo. Un año después del peak de ideación suicida -esto es, a los 16 años-, se presentó la mayor incidencia de intento suicida (14\%), la cual disminuyó a los 17 y 18 años (ambos 5\%).

Al comparar la ideación e intento suicida según tipo de colegio (Figura 5) se observó que la prevalencia de ideación suicida en colegios municipales $(65 \%)$ y subvencionados $(67 \%)$ no tiene diferencias significativas $(\mathrm{p}=0,058)$, mientras que en los colegios privados la prevalencia de ideación suicida (39\%) sí es significativamente menor $(\mathrm{p}<0,001)$. La prevalencia de vida de in- 


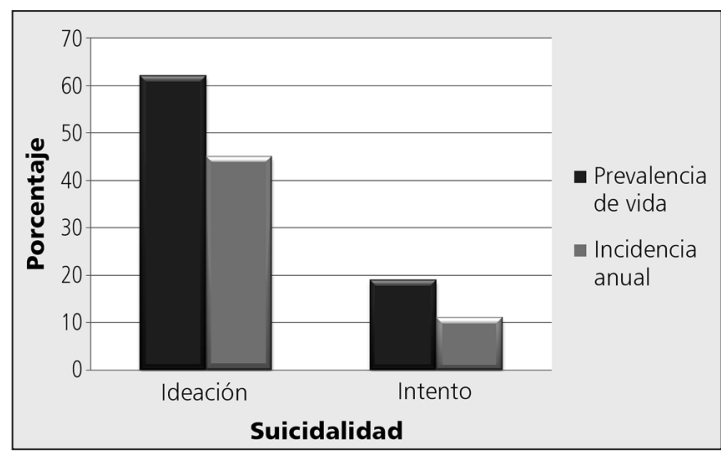

Figura 1. Prevalencia de vida e incidencia anual de ideación e intento suicida en población adolescente de la Región Metropolitana (RM), Santiago (Chile), 2007. $(n=1.249)$.

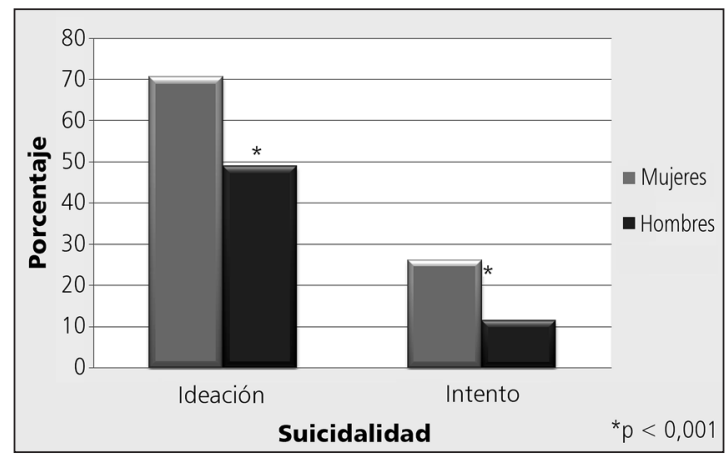

Figura 3. Prevalencia de vida de ideación e intento suicida en adolescentes de la Región Metropolitana (RM) según sexo, 2007.

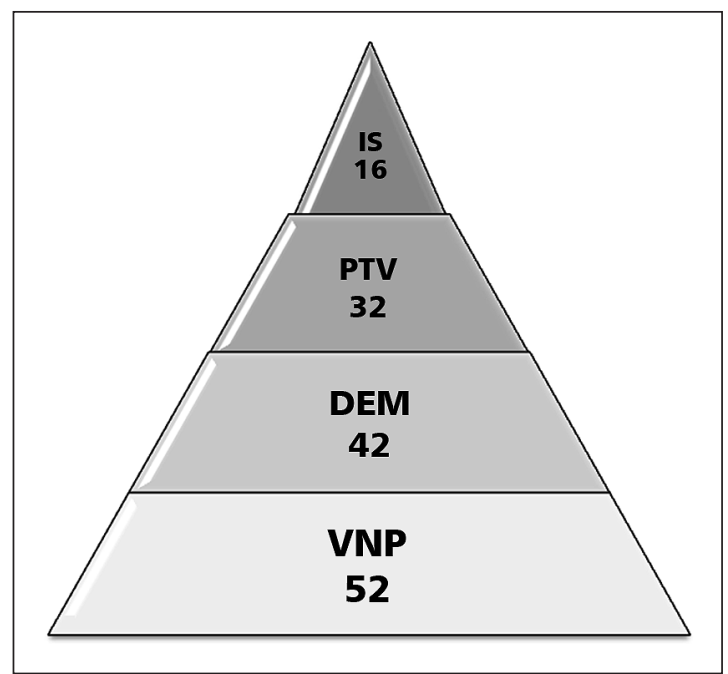

Figura 2. Pirámide que establece una relación numérica entre la distinta gravedad de la suicidalidad. VNP: La vida no vale la pena; DEM: Ha deseado estar muerto; PTV: Ha pensado en terminar con la vida; IS: Intento suicida.

tento suicida en los colegios municipales alcanzó a $29 \%$; en los subvencionados, a $18 \%$; y en los privados, a $4,7 \%$, todas con diferencias estadísticamente significativas $(\mathrm{p}<0,001)$. En todos los tipos de colegio se observó un descenso en la prevalencia a medida que aumenta la gravedad de la suicidalidad.

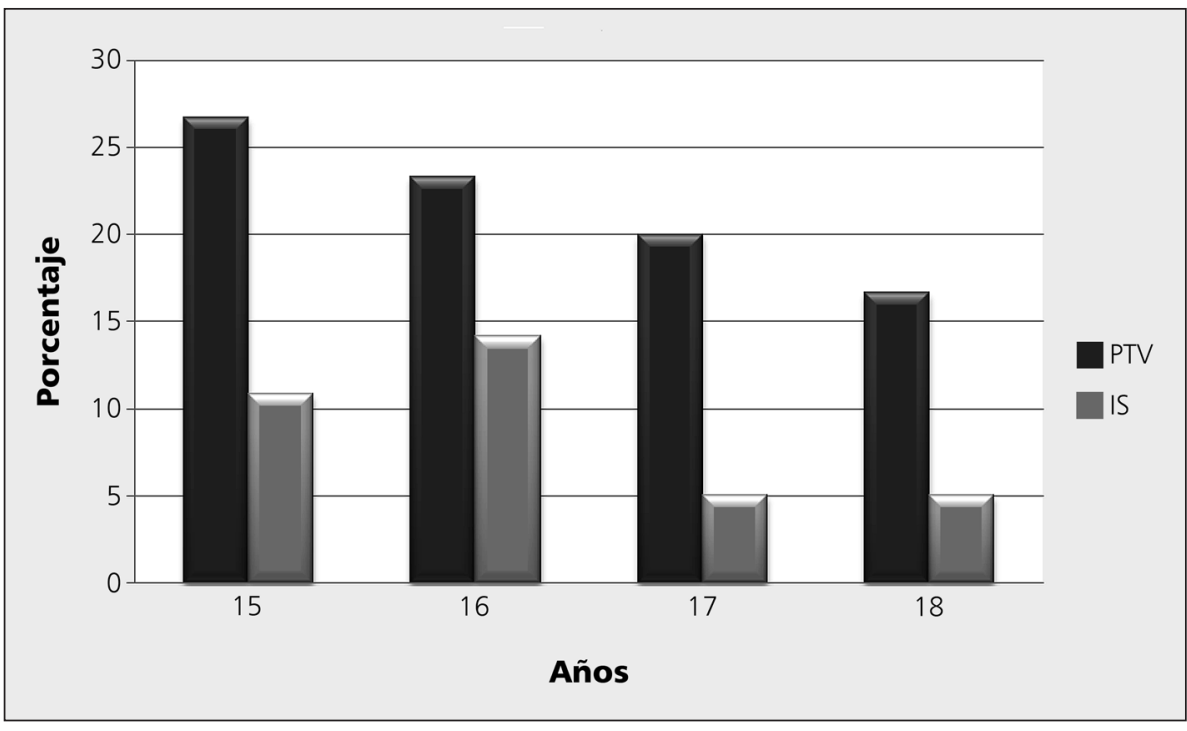

Figura 4. Prevalencia de vida de ideación e intento suicida en adolescentes de la RM según edad, 2007. PTV: Ha pensado en terminar con la vida, IS: intento suicida. 


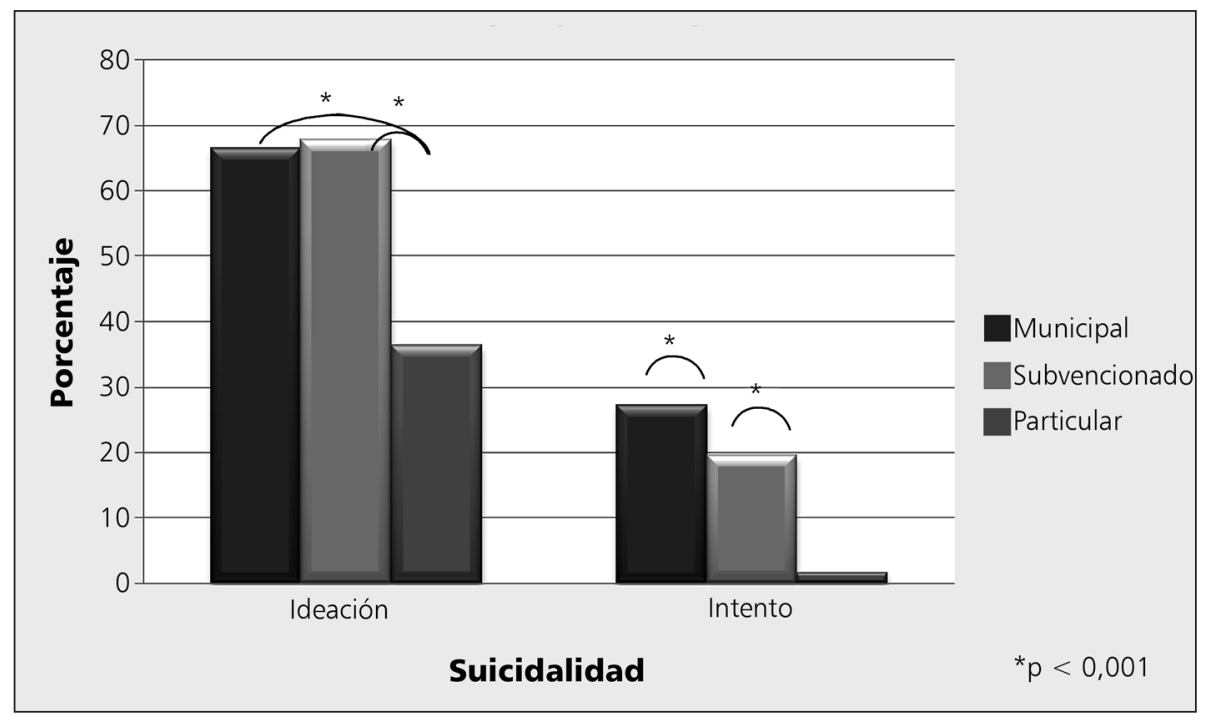

Figura 5. Prevalencia de vida de ideación e intento suicida en adolescentes de la RM según tipo de colegio, 2007

\section{Discusión}

Los resultados obtenidos en este estudio son similares en muchos aspectos a los de la literatura revisada. Al comparar los resultados de prevalencia de ideación $(59,7 \%)$ e intento suicida $(18,8 \%)$ en adolescentes de la RM con trabajos anteriores hechos en Chile, destaca la gran similitud entre ellos. En un trabajo sobre el mismo tema realizado por Salvo et $\mathrm{al}^{5}$ en Concepción, la prevalencia encontrada para ideación es de $57 \%$ y de $14,2 \%$ para intento, observándose un leve aumento en nuestros valores. La similitud entre los valores de prevalencia e incidencia anual para ideación (62 vs 45) e intento (19 vs 11) puede entenderse porque el fenómeno de la suicidalidad se inicia preferentemente a esta edad. Otro estudio de depresión y suicidalidad en población no clínica de adolescentes de Calama efectuado por Quinteros et $\mathrm{al}^{22}$, que tiene una muestra muy parecida en su distribución por sexo y edad a la nuestra y que empleó el mismo instrumento, describe una prevalencia de PTV de 30,5\%, mientras la nuestra es de $36 \%$. En otro estudio chileno, de Valdivia et $\mathrm{al}^{23}$, se observa $14,2 \%$ de prevalencia de vida para los intentos suicidas, $4,4 \%$ menos que el presente estudio. Al compararlo con literatura extranjera, se observa una gran diferencia en las cifras, donde Chile supera con creces los valores en ideación e intento suicida. Un estudio prospectivo sobre la historia natural del suicidio en jóvenes en Canadá de Brezo et $\mathrm{al}^{7}$, muestra una prevalencia de $33 \%$ de ideación suicida y $9,3 \%$ de intento. Un estudio realizado en Italia para evaluar ideación suicida y agresividad en jóvenes escolares observa una prevalencia de ideación suicida de sólo 10,5\%, bastante inferior a esta investigación ${ }^{24}$ Sin embargo, los resultados de este último estudio son menos comparables debido a diferencias socioculturales y metodológicas.

$\mathrm{Al}$ igual que en el trabajo de Salvo et $\mathrm{al}^{5}$, la mayor prevalencia de suicidalidad se presenta en los niveles más leves de ideación suicida (VNP y DEM). Existe una superposición entre diferentes niveles de suicidalidad, donde los rangos de mayor gravedad también incluyen niveles de suicidalidad menos graves. El intento suicida se asocia significativamente a niveles elevados de ideación suicida, principalmente PTV.

En cuanto a las diferencias por sexo, se observa una clara prevalencia de suicidalidad en mujeres. Del total de ellas, $71 \%$ ha tenido ideación suicida y $26 \%$ ha intentado quitarse la vida. Con respecto a los hombres, $49 \%$ del total ha presentado ideación suicida y $12 \%$ ha cometido un intento de suicidio. Según lo postulado por Valdivia et al $^{23}$, la relación de intentos suicidas entre hombres y mujeres es de 3 a 1; relación que es concordante con nuestro trabajo donde las mujeres tienen cuatro veces más intentos que los hombres. 
En la distribución de suicidalidad entre las distintas edades se observa que la incidencia más alta de ideación grave (PTV) ocurre a los 15 años, un año antes que la mayor frecuencia de intentos suicidas (16 años).

Al comparar los resultados por tipo de colegio (municipal, subvencionado y privado), que podría considerarse como la variable socioeconómica, se observa una marcada diferencia entre los alumnos de los colegios municipales y subvencionados versus aquellos de los privados. Entre los dos primeros tipos de colegio no hay una clara diferencia entre ideación suicida; sin embargo, el intento es más frecuente en colegios municipales que en los subvencionados. La ideación es bastante mayor en los colegios municipales $(66,3 \%)$ y subvencionados $(69,4 \%)$ que en los privados $(46,3 \%)$. Los factores de riesgo que subyacen en estas diferencias escapan al objetivo de este estudio pero son múltiples como se observa en el estudio danés de Ping Qin ${ }^{25}$, entre las que destacan los antecedentes familiares de suicidio y enfermedad mental. Estos valores son preocupantes ya que del total de alumnos matriculados en colegios de Chile, $48 \%$ están en establecimientos municipales, $43 \%$ en subvencionados y sólo $7 \%$ en privados.

\section{Referencias}

1. OMS. Prevención del suicidio: Un instrumento para docentes y demás personal institucional [monografía en internet]. Ginebra; OMS; 2001. [acceso 12 de Noviembre 2007]. Disponible en: http://www.who.int/ mental_health/media/en/63.pdf.

2. Lester D. Adolescent suicide risk today: A paradox. J Adolesc 1998; 21: 499-503.

3. Mittendorfer-Rutz E. Trends of youth suicide in Europe during the 1980s and 1990s-gender differences and implications for prevention. J Mens Health Gend 2006; 3: 250-7.

4. Weil K. Urgencias psiquiátricas. En: Florenzano R, Carvajal C, Weil K. Psiquiatría. Santiago, Chile: Mediterráneo Ltda.; 1999. p. 216-27.

5. Salvo L, Rioseco P, Salvo S. Ideación suicida e intento de suicidio en adolescentes de enseñanza media. Rev Chil Neuropsiquiat 1998; 36: 28-34.

6. Haquim C, Larraguibel M, Cabezas J. Factores protectores y de riesgo en salud mental en niños y adolescentes de la ciudad de Calama. Rev Chil Pediatr 2004; 75: 425 33.
7. Brezo J, Paris J, Barrer E. Natural history of suicidal behaviors in a population-based sample of young adults. Psychol Med 2007; 37: 1563-74.

8. Waldrop AE, Hanson RF, Resnick HS, Kilpatrick DG, Naugle AE, Saunders BE. Risk factors for suicidal behavior among a national sample of adolescents: Implications for prevention. J Trauma Stress 2007; 20: 869-79.

9. Evans E, Hawton K, Rodham K, Deeks J. The prevalence of suicidal phenomena in adolescents: a systematic review of population-based studies. Suicide Life Threat Behav 2005; 35: 239-50.

10. Steele M, Doey T. Suicidal behaviors in Children and Adolescents. Part 1 Etiology and risk factors. Can J Psychiatry 2007; 52 (6 Suppl 1): 21S-33S.

11. De Leo D, Cerin E, Spathonis K, Burgis S. Lifetime risk of suicide ideation and attempts in an Australian community: Prevalence, suicidal process, and help-seeking behavior. J Affect Disord 2005; 86: 215-24.

12. Fortune S, Hawton K. Suicide and deliberate self-harm in children and adolescents. Paediatr Child Health 2007; 17: 443-7.

13. Prada DB, Evangelista M, Piola JC. Tentativas de suicidio con tóxicos en adolescentes en Rosario, Argentina. Braz Pediatr News. 2002; 4. Disponible en: http://www.brazilpednews.org.br/dec2002/bnp4003.htm [Consultado el 26 Octubre 2007].

14. Serfaty E. Suicidio en la adolescencia. Adolesc Latinoam 1998; 1 (2): 105-10.

15. Ulloa F. Tentativas y consumación de suicidio en niños y adolescentes. Rev Chil Pediatr 1993; 64: 272-6.

16. Rona E, Wettlin L. Tentativas de suicidio en niños $y$ adolescentes. Rev Chil Pediatr 1986; 57: 227-30.

17. Larraguibel M, González P, Martínez V, Valenzuela R. Factores de riesgo de la conducta suicida en niños y adolescentes. Rev Chil Pediatr 2000; 71 (3): 183-91.

18. Zung WW. A self rating depression scale. Arch Gen Psychiatr 1965; 12: 63-70.

19. Okasha A, Lotaif F, Sadek A. Prevalence of suicidal feelings in a sample of non-consulting medical students. Acta Psychiatr Scand 1981; 63: 409-15.

20. Salvo L, Melipillán R, Castro A. Confiabilidad, validez y punto de corte para escala de screening de suicidalidad en adolescentes. Rev Chil Neuro-Psiquiatr 2009; 47: 1623.

21. Mineduc [base de datos en Internet]. Santiago, Chile; 2003- [actualizado el 30 de abril de 2006; acceso 13 de noviembre de 2007]. Tabla de matrícula año 2006. Disponible en: w3app.mineduc.cl/mineduc/ded/documentos/Matricula\%202006.zip

22. Quinteros P, Grob F. Depresión y suicidalidad en una 
Prevalencia de ideación e intento suicida en adolescentes de Santiago de Chile - R. Ventura-Juncá D. et al

población no clínica de adolescentes. Bol Soc Psiquiat Neurol Infanc Adolesc 2003; 14: 4-8.

23. Valdivia M, Schaub C, Díaz M. Intento de suicidio en niños: algunos aspectos biodemográficos. Rev Chil Pediatr 1998; 64-7.

24. Miotto P, De Coppi M, Frezza M, Petretto D, Masala C,
Preti A. Suicidal ideation and aggressiveness in schoolaged youths. Psychiatry Res 2003; 120: 247-55.

25. Ping Q, Agerbo E, Mortensen PB. Suicide risk in relation to family history of completed suicide and psychiatric disorders: a nested case-control study based on longitudinal registers. Lancet 2002; 360: 1126-30. 